\title{
TRES SOLUCIONES EN LA RESTAURACIÓN DEL PALACIO DE TORAL DE LOS GUZMANES. LEÓN-ESPAÑA
}

\author{
(THREE SOLUTIONS IN THE RESTAURATION OF THE PALACIO DE TORAL DE LOS \\ GUZMANES. LEÓN-SPAIN)
}

Eloy Algorri García, Arquitecto.

Fecha de recepción: $21-\mathrm{X}-94$

128-61

\section{RESUMEN}

El Palacio de Toral de los Guzmanes es un edificio cuyos muros están predominantemente construidos con fábrica de tapia. Uno de los objetivos básicos de la restauración, que viene realizándose desde 1985, es el progreso en el conocimiento de esta fábrica tan común en la arquitectura monumental española. Tras unas reflexiones de indole general, el autor establece un decálogo abreviado del comportamiento constructivo y resistente de la tapia y lo ejemplifica con la descripción y el análisis de tres soluciones a problemas específicos que se han experimentado en el curso de las obras.

\section{SUMMARY}

The Palacio de Toral de los Guzmanes is a building whose walls are mostly made of "tapia" (rammed earth). One of the basic objetives of the restauration which began in 1985 is acquiring new information in order to extend the knowledge of this type of masonry, commonly used in Spanish monumental architecture. After a few general considerations the author establishes an abbreviated decalogue of construction and strength behaviour of the "tapia". The example used as an illustration is that of the description and analysis for three solutions to specific problems which appeared during the restauration works.

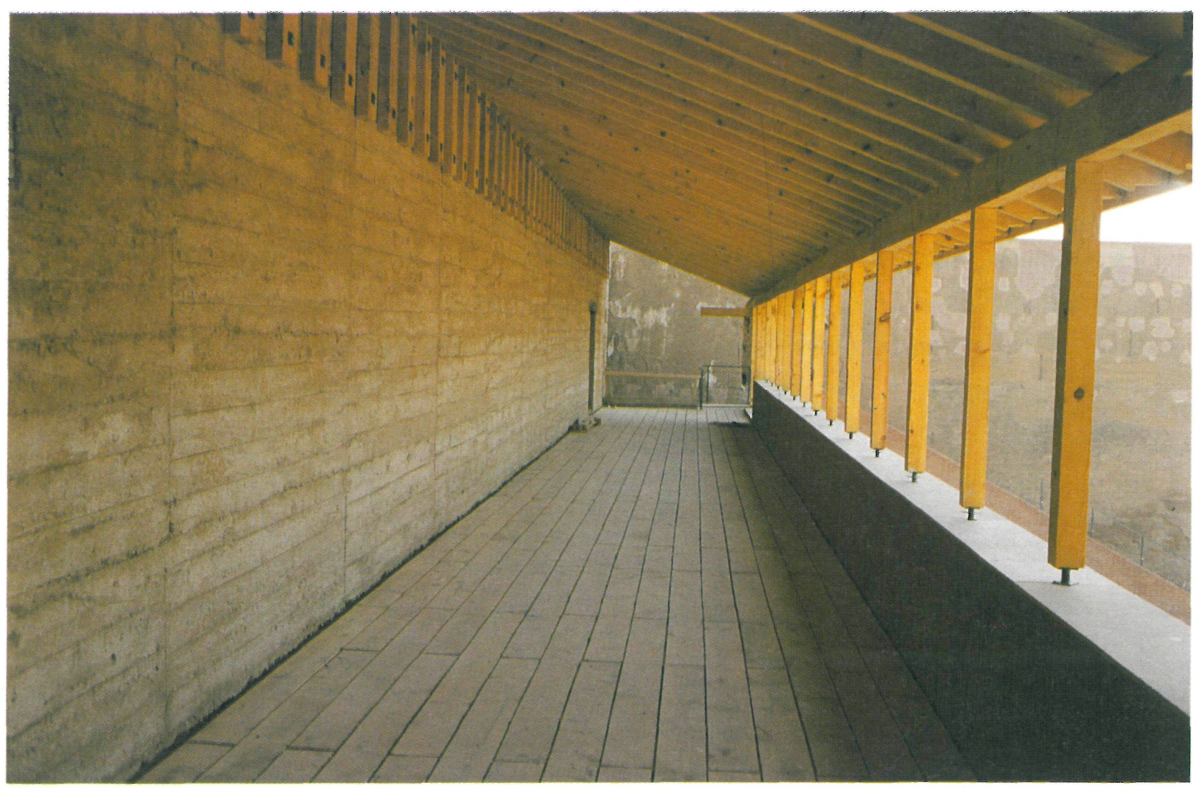

La planta primera de la galeria porticada. 
En 1985 se emprendió la restauración del Palacio de Toral de los Guzmanes con la intención de invertir el proceso ruinoso que, incluso, amenazaba la desaparición del edificio en un plazo no muy $\operatorname{largo}^{1}$.

Este objetivo concreto implicaba simultáneamente la posibilidad de satisfacer otro de índole mucho más general: la profundización en el conocimiento de las particularidades de la tapia, fábrica con la que está construida la mayor parte de los muros del edificio.

De este modo, la restauración se orientó en un sentido experimental cuya utilidad rebasara sus propios límites y sirviera para afrontar con mayor conocimiento de causa posteriores intervenciones sobre una fábrica, tan común en el patrimonio monumental de nuestro país como desconocida para la generalidad de las distintas profesiones intervinientes en el campo de la restauración.

En otro lugar ya hemos dado cuenta pública de las vicisitudes y enseñanzas de la parte más espectacular de la restauración del Palacio de Toral consistente en la erección, con técnicas y medios auxiliares modernos, de muros de tapia de gran espesor y altura notable ${ }^{2}$.

No obstante, tal vez no sea éste el capítulo que pueda resultar de mayor utilidad. La reconstrucción total o parcial de muros de tapia no dejará nunca de ser una operación excepcional, restringida generalmente a antiguas edificaciones de carácter militar donde la tapia tendió a utilizarse como fábrica exclusiva.

Sin embargo, a lo largo de estos años hemos constatado en congresos, publicaciones y otros medios de difusión, la considerable frecuencia con la que se producen intervenciones muy diversas sobre muros de tapia ya existentes. Esto no es extraño, si consideramos la enorme profusión de la tapia en la arquitectura monumental de nuestro país, la mayor parte de las veces como fragmento intercalado con otras fábricas.

Desgraciadamente hemos detectado, en la mayoría de estos casos, una general desorientación de los profesionales encargados que a todas luces desconocen y, lo que es peor, desconfían de las capacidades del material.

No creemos que el remedio a este vacío sea la creación de una casta de especialistas de la tapia que engrose el creciente colegio sacerdotal y que, irremediablemente, acompaña toda restauración con pretensión "científica". Por el contrario, es la tapia una fábrica tan elemental en sus principios constructivos que resulta perfectamente asequible a cualquier profesional interesado.

Esta intención difusora es la que respalda el presente artículo. Nos centraremos, por tanto, en aquellos aspectos de la restauración del Palacio de Toral, relativos a las fábricas de tapia que consideramos de mayor utilidad, es decir, a la casuística más común de intervención en tapias ya existentes.

Por este motivo, vamos a centrarnos en la descripción y el análisis de algunas soluciones experimentadas en la restauración del Palacio de Toral, unas más afortunadas y otras menos, con el objetivo no tanto de establecer un canon, sino de ayudar a reflexionar sobre el carácter de la tapia y sus exigencias específicas.

Por claridad pedagógica, antes de pasar a la parte descriptiva, hemos de realizar una previa exposición de los principios constructivos básicos de los muros de tapia.

Consideramos que ésta es una imprescindible introducción, pues para afrontar la restauración de un edificio es necesaria una tarea de comprensión del esquema lógico manejado por aquellos que lo levantaron. O dicho de otro modo más tajante: hay que entender el edificio como si uno fuera a realizarlo de nuevo.

La cuestión estriba en que los cambios sociales que se han operado en el presente siglo y las consecuencias derivadas (división del trabajo, utilización de nuevos recursos y materiales, etc.) han supuesto un salto que quiebra la continuidad en la evolución de las técnicas de la construcción y, lo que es más importante, en la forma de concebir el proceso edificatorio. Es preciso, por tanto, despojarnos de los lugares comunes de la construcción contemporánea (tarea difícil, por cierto, pues es algo que inconscientemente arrastramos) y reencarnarnos de la forma de pensar de nuestros ancestros.

A su vez, entendemos que la restauración intenta reponer a un valor adecuado aquellas propiedades que el edificio ha ido perdiendo por envejecimiento. Este fenómeno se produce habitualmente por falta de conservación, si bien en ocasiones tal labor no es posible con un esfuerzo razonable, pues el proyecto original no atendió adecuada- 
mente a la durabilidad del edificio. Sería jocoso que la restauración recayera en lo mismo, dejando nuevo el edificio aunque sin posibilidad de conservarlo o con vicios ocultos.

Por tanto, hay que entender los modos específicos en los que cada técnica responde a los requisitos constructivos y cómo cada material impone límites a través de las exigencias de durabilidad. En el caso de la tapia, estos límites son excepcionalmente exigentes.

Por esta razón el objetivo primero será comprender los principios que rigen la lógica de la construcción con tapia, considerando que algunos de estos conceptos son comunes a todas las antiguas técnicas de edificación y otros son específicos del material que nos ocupa como consecuencia de sus particulares propiedades.

La naturaleza constructiva de los muros de tapia puede resumirse en los siguientes conceptos básicos:

- Homogeneidad: tal vez lo más significativo de la evolución técnica contemporánea sea la difusión generalizada de materiales monofuncionales (portantes, aislantes, impermeabilizantes), de modo que los distintos elementos constructivos se resuelven como heterogénea suma de éstos. Por el contrario, en el caso presente, un solo material - la tierra compactada- obtiene, a través del espesor, unos niveles de capacidad portante, conductividad e inercia térmica, estanquidad, etc., con los que resuelve satisfactoriamente los requerimientos que ha de afrontar. En consecuencia, frente al principio de libertad de movimientos para cada una de sus partes (que tienen comportamientos térmicos, resistentes, etc., muy diferentes), en los que se basa la construcción moderna, el objetivo ha de ser justamente el contrario: la sólida trabazón del conjunto.

- Independencia de las partes: en realidad esta trabazón total es inalcanzable pues los distintos lienzos de una construcción de tapia tienden, por la escasa resistencia de la fábrica a esfuerzo cortante y a tracción, a independizarse por rotura vertical allí donde el muro es más débil, es decir, en todos los planos verticales transversales a la pared donde coinciden las juntas de construcción, especialmente en las esquinas.

- Autoestabilidad: los muros de tapia son en general de un generoso espesor, por lo que tie- nen habitualmente esbelteces pequeñas, nunca superiores a 1-10 entre espesor y altura, y una fuerte componente de peso propio, de modo que tan sólo en circunstancias excepcionales se puede correr el peligro de que la resultante de las acciones no pase por el núcleo central de inercia. Es decir, "cada fragmento vertical del mismo es capaz de sostenerse y de colaborar en la estabilidad general del edificio... Es un sistema que funciona por simple adición de parámentos" 3 . Por tanto, la pérdida de trabazón que se produce como consecuencia de la tendencia de la fábrica a la fragmentación queda compensada por la estabilidad de cada una de las partes en las que se descompone. "Por ello, no suelen ser preocupantes las fisuras verticales..., la diferenciación de estas piezas por fisuras de retracción, dilatación térmica o asiento diferencial no tienen necesariamente... graves consecuencias" ${ }^{4}$. Afirmación que hay que matizar pues si bien es cierto que la estabilidad del conjunto inicialmente no se resiente, el agrietamiento afecta a medio plazo a la durabilidad de los muros por la desprotección que representan estos puntos de rotura respecto de los agentes agresivos. El muro no se desploma, pero poco a poco se desmorona.

A su vez, lo esencial del comportamiento estructural de los muros de tapia puede resumirse en breves líneas:

- Las fábricas de tapia están concebidas para resistir exclusivamente esfuerzos de compresión simple.

- En los planos más solicitados, es decir las secciones horizontales inferiores, la parte mayoritaria de los esfuerzos que soporta es la debida al peso propio de la fábrica que como resulta obvio es más relevante cuanto más alto es el muro. Habitualmente la carga viva no supera el 15 por ciento. En resumen, el muro que está en pie resistirá sin problemas el peso derivado de nuevos forjados o cubiertas cuyas sobrecargas no excedan de lo habitual.

- La tensión correspondiente al peso propio puede cifrarse en torno a los $0,02 \mathrm{mN} / \mathrm{m}^{2}$ por cada metro de altura del muro, como corresponde a la densidad del material cuyo valor medio aproximado es de $2.000 \mathrm{~kg} / \mathrm{m}^{3}$.

- Es prácticamente imposible agotar la resistencia a compresión simple de la tapia. En concreto, la 
tensión de rotura de una tapia tradicional de calidad media sin aditivos aglomerantes oscila de $1 \mathrm{a} 1,4 \mathrm{mN} / \mathrm{m}^{2}$ lo que equivale a un tamaño insuperable de $50 \mathrm{~m}$ de altura.

- La calidad de todas las fábricas de tierra es heterogénea como consecuencia de la naturaleza de la materia prima (en la mayoría de los casos la tierra se utiliza tal como sale sin correcciones posteriores) y las visicitudes de la puesta en obra, que se rige habitualmente por normas ambiguas aplicadas de forma subjetiva. Por tanto, los criterios para determinar los valores de la tensión segura han de ser muy prudentes, aplicando unos coeficientes de seguridad altos. Guiándonos con la Norma Básica del Ladrillo estimamos que 3 puede ser un coeficiente de minoración razonable. En este caso la tensión segura de la tapia sería de $0,4 \mathrm{mN} / \mathrm{m}^{2}$ (equivalente a $20 \mathrm{~m}$ de altura). Es decir, al contrario que en las estructuras convencionales, los muros de tapia están bastante cerca de su tamaño insuperable seguro.

- Además, las fábricas de tierra tienen respecto de otras una particularidad esencial: su capacidad resistente puede variar enormemente en un lapso de tiempo muy breve por distintas causas patológicas. Es decir, a pesar de que los números que hemos manejado aportan una sensación de seguridad, es necesario tener en cuenta que hay factores que pueden producir un rápido descenso de estos valores. Entre ellos destacamos dos:

- La vulnerabilidad del material ocasiona una rápida erosión de los muros especialmente en los puntos más delicados, entre los que destaca el zócalo, donde convergen las agresiones mecánicas y la acción destructiva del agua ya sea por capilaridad, salpicadura u otros factores. Esto produce una disminución de la superficie de la sección horizontal que precisamente es la más solicitada.

- La capacidad resistente de la fábrica disminuye en proporción geométrica a la cantidad de agua que retiene en su interior. Avisamos que tras este envoltorio tan tajante sólo hay una hipótesis que se funda, eso sí, en un hecho comprobado. Al construir un muro de tapia nuevo, la fábrica va ganando resistencia en la medida que va secando. Mientras el muro retiene el agua de amasado su resistencia es muy escasa, con la particularidad de que una pequeña cantidad de agua produce una disminución importantísima de la capacidad resistente. Para hacerse una idea numérica, una tapia que seca resiste $1 \mathrm{mN} / \mathrm{m}^{2}$ (tensión de rotura) con una cantidad de agua no superior en peso al 10 por ciento de la masa total ni siquiera admite una tensión de 0,1 $\mathrm{mN} / \mathrm{m}^{2}$.

Lo que no sabemos es (por eso decimos que es una hipótesis) si este fenómeno es reversible, es decir, si una tapia que eliminó la humedad incorporada al proceso de construcción absorbe agua, pór la razón que sea, sufre una pérdida de resistencia del mismo calibre. En principio, al tratarse de fábricas cohesionadas con aglomerantes donde no se produce una metamorfosis cualitativa, la reversibilidad parece lo más razonable; sin embargo, en la realidad se observan muchos muros que según esta ley no deberían mantenerse en pie.

- La tapia es un material muy frágil. Es decir, el agotamiento de la capacidad resistente no está anticipado por deformaciones previas. La superación de su resistencia a cortante y a tracción ocasiona un agrietamiento vertical y el colapso súbito a compresión simple.

Sentadas estas bases, vamos a ejemplificarlas mediante el desarrollo concreto de las soluciones que adoptamos en tres de los muchos problemas que se han planteado en el curso de la restauración del Palacio de Toral.

\section{Conservación de un muro de tapia sin resguardo}

El criterio que se adoptó como pauta general de intervención fue la restitución arquitectónica del conjunto. Consideramos que la mejor manera de conservar los fragmentos supervivientes era reintegrarlos como partes de un edificio nuevo que reprodujera las características constructivas y tipológicas del precedente y conservara sus valores documentales.

Esta operación reconstructora, que podría resultar ilegal en los términos establecidos en la vigente Ley del Patrimonio Histórico-Artístico, nos pareció sin embargo la única respuesta razonable a las particulares exigencias de las fábricas de tapia. 


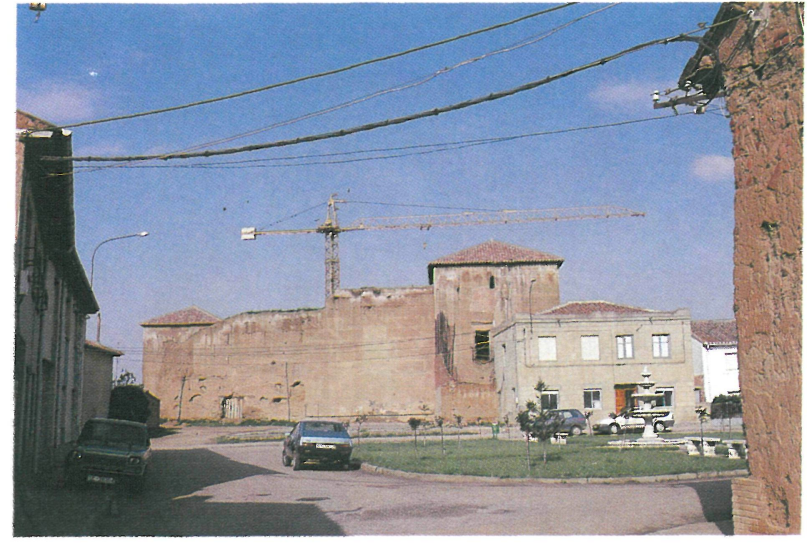

Foto 1. Tapia sin resguardo antes de la restauración.

En efecto, la gama de intervenciones clásicas, según los distintos cánones de la restauración (de la limpieza a la anastilosis), se derivan directamente de las características de la arquitectura pétrea que, en un estado incompleto o aparentemente ruinoso, es capaz de resistir aceptablemente la acción agresiva de los agentes atmosféricos.

Ocurre que éste no es el caso de los muros de tapia. Llegados a este punto, sugerimos la conveniencia de ampliar la gama de intervenciones canónicas incluyendo aquellas que resulten idóneas para las fábricas deleznables, pero éste ya es tema de otro artículo.

Volviendo a lo que nos ocupa, la mayoría de los antiguos lienzos del Palacio de Toral se preservan indemnes mediante su integración en un nuevo edificio completo con el que se recuperan los mecanismos habituales de conservación y, especialmente entre ellos, el cobijo de una cubierta.

Sin embargo, por razones que escapan del alcance de este artículo, uno de los lienzos ha quedado inevitablemente fuera de esta operación de resguardo. Hemos debido, por tanto, plantear su conservación desde parámetros no reconstructores.

El lienzo tiene una longitud aproximada de $25 \mathrm{~m}$, un espesor de 2,3 y una altura variable, según un perfil irregularmente escalonado, con una cota máxima de $12 \mathrm{~m}$.

Constructivamente se compone de un zócalo de mampostería de 2 metros de altura aproximada y una fábrica de tapia calicostrada, es decir, tierra compactada y revestida en sus paramentos con un mortero de cal aplicado al mismo tiempo que se procede a la erección del muro ${ }^{5}$.

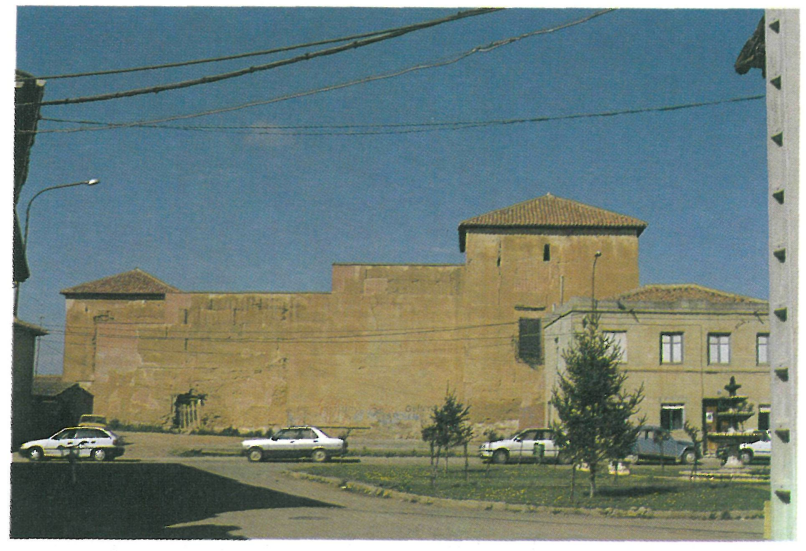

Foto 2. Tapia sin resguardo después de la restauración.

El dispositivo de conservación previsto en el proyecto original ${ }^{6}$, frente a la acción del agente agresivo por antonomasia - el agua atmosférica-, es una protección impermeable compuesta por el zócalo de mampostería, el calicostrado de los paramentos y un elemento de cobertura superior. Por tanto, el muro no posee una envolvente estanca continua sino que depende en su plano horizontal de coronación de la existencia de un elemento ajeno. Éste es su talón de Aquiles.

Los daños que presentaba el muro en el momento de la restauración eran básicamente de tres tipos, aunque siempre derivados de la rotura de la envolvente impermeable:

- Desconchados en uno de los paramentos donde, por motivos diversos, generalmente la apertura de mechinales de vigas, se había roto el calicostrado.

- Desmoronamiento de la parte superior de la fábrica ocasionada por la infiltración del agua.

- Desmoronamiento del muro en el plano transversal, que ha quedado expuesto a la acción del agua debido a la desaparición de los elementos arquitectónicos vecinos.

La solución es conceptualmente elemental y ha de consistir obviamente en la redefinición de una nueva envolvente impermeable exenta de discontinuidades.

Para rehacer el paramento calicostrado hemos seguido las pautas que marcaban reparaciones precedentes. Consistían éstas en remendar la superficie deteriorada con plastones de fábrica de ladrillo rojo. 


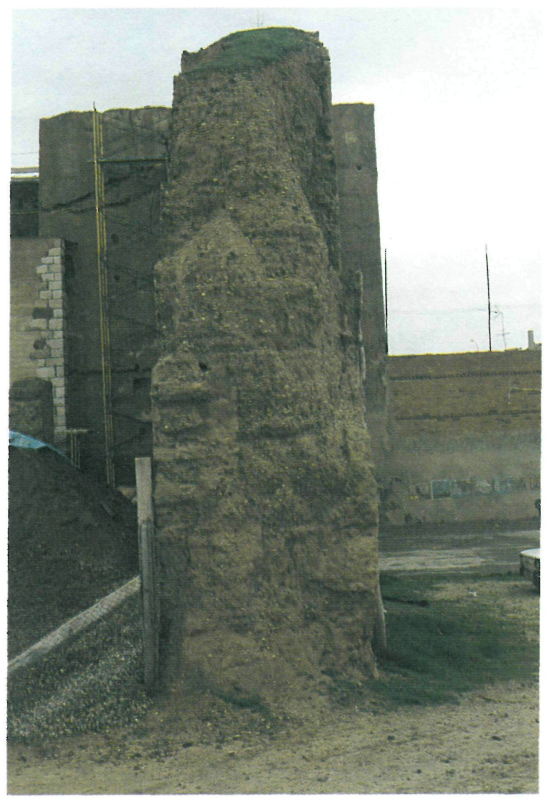

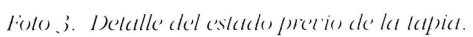

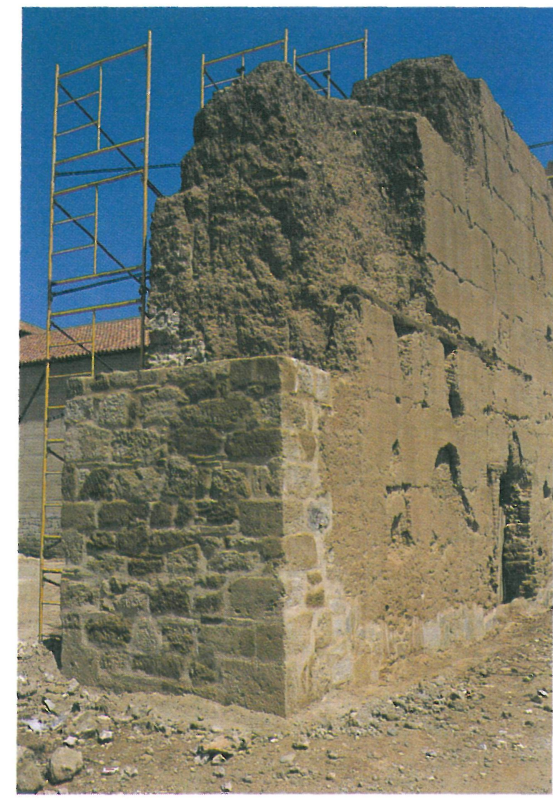

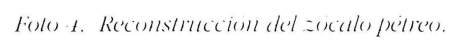

Dos son las razones por las cuales hemos dado por buena esta solución.

En primer lugar, consideramos que una de las características esenciales de la tapia consiste en ser un material formáceo en cuya superficie se dibuja no sólo el negativo del molde, sino también todo el proceso de puesta en obra. Por este motivo desechamos cualquier solución de tendido, es decir de revestimiento de mortero que en ningún caso podrá reproducir la expresividad del calicostrado. Frente a la inalcanzable mímesis hemos preferido el contraste visual y la semejanza conceptual con otra fábrica "desnuda".

Por otra parte, la reconstrucción de la cubierta y de las dos alas del patio otorgan una fortísima presencia a la cerámica roja que se convierte en un material con un peso casi equiparable al de la tapia, por otra parte, tal como ocurría en el Palacio en su estado original.

La puesta en obra de la reparación de los desconchados se ha realizado siguiendo las leyes de la trabazón, con objeto de que estos añadidos queden sólidamente unidos al conjunto de la fábrica.

Para ello, se ha utilizado como elemento de engarce entre tapia y ladrillo una masa de hormigón pobre que se vierte entre ambos. La adherencia de éste y la tapia se consigue mediante la penetración de la masa en los recovecos de la pared deteriorada. El ladrillo es perforado y se apareja, formando un encofrado perdido, al modo flamenco, dejando los tizones completos para que sirvan de llaves de conexión con el hormigón.

Esta solución, que es técnicamente irreprochable, produce un impacto excesivo de contraste en la imagen del lienzo si la superficie a reparar es porcentualmente cuantiosa, sobre todo cuando la iluminación solar es rasante. Para estos casos particulares dudamos en utilizar, excepcionalmente un ladrillo más entonado con la tapia, pero finalmente optamos por ajustarnos a la limitada restricción de materiales, texturas y colores que nos habíamos impuesto al comienzo de la restauración.

En la disyuntiva entre los conceptos y la imagen nos decantamos por aquello que consideramos esencial. No obstante, hay que reconocer que esperamos como agua de mayo la acción benéfica de lluvias, chaparrones y tormentas que a lo largo de los años vayan lavando los lienzos, tiñéndolos de un homogéneo tono terroso que unifique tapia, piedra y ladrillo, tal como sucedió con reparaciones precedentes.

La continuidad de la envolvente impermeable en las coronaciones y la sección transversal se abordó de una manera conjunta. Para ello, se decidió regularizar la silueta escalonada del muro según una traza descendente acorde con nuestra sensibilidad compositiva y que, a la vez, implicará el menor volumen de obra posible. 
El perfil elegido requirió la construcción de un cierto volumen de fábrica de tapia a una sola cara y, en algún punto particular, una pequeña demolición parcial de la antigua.

Esta tapia nueva se realizó para reparar todos los desconchados y desmoronamientos que, por su posición en la parte alta del muro, admitían la posibilidad de ejecutar el apisonado.

El escalonamiento elegido descompuso la sección transversal en cuatro planos. Los tres superiores, que corresponden con la franja del muro construida en tapia, se han rematado con unas piezas de fábrica de ladrillo a modo de incrustaciones en forma de L invertida, que expresan en su trazado las alturas de las hiladas de la tapia. Estos remates de albañilería siguen las leyes de trabazón y aparejo descritas con anterioridad.

El último peldaño coincide con el zócalo de mampostería y se construye en esta misma fábrica con un afán de mímesis en la observación de conjunto y de leve diferencia en la de detalle.

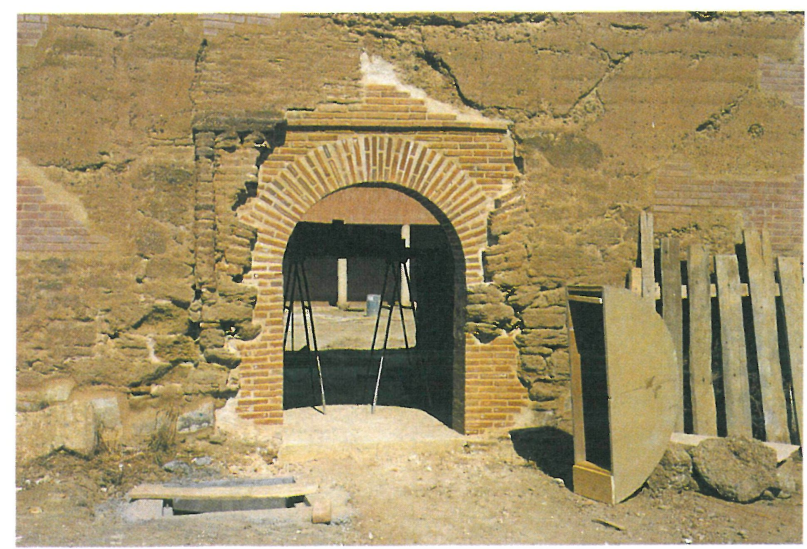

Foto 5. Restauración de un elemento singular.

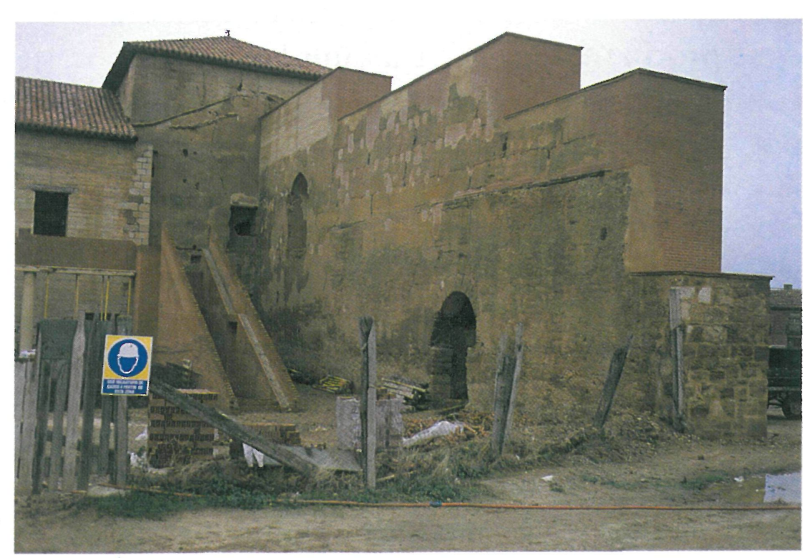

Foto 6. La tapia restaurada desde el interior del patio.
La solución de perfil escalonado convierte la coronación en una sucesión de planos horizontales, geometría que en principio no es muy favorable a la evacuación de las aguas.

Desechamos cualquier solución de tejadillos que, aunque muy eficaz, tiene una impronta doméstica y pintoresca impropia del carácter y dimensiones del edificio.

Consideramos que una albardilla de dos capas casi impermeables bastaría para completar la envolvente estanca. En consecuencia se remató el muro con una liviana losa de hormigón armado recubierta con un enlosado de piezas cerámicas, ligeramente inclinado a dos aguas, rematado en su perímetro con una minúscula cornisa que hace de goterón.

Aunque podrían haberse utilizado materiales, que con bajas pendientes son más impermeables que la cerámica, nos sometimos de nuevo a la previa selección de materiales. Influyó además en este caso la importancia de la unidad en la imagen aérea del Palacio.

\section{Apoyo sobre un muro de tapia de un forjado de hormigón armado}

La habilitación de las naves del Palacio para alojar funciones que lo revitalicen ha requerido la construcción de forjados de nueva planta.

Quedaban restos que atestiguaban suficientemente cómo eran los originales: una familia principal de vigas de gran escuadría de roble o chopo, separadas entre sí metro y medio, sobre las que se colocaba un entablado, con el concurso previsible de una familia intermedia de correas.

Aunque en la erección de las nuevas fábricas se habían previsto los oportunos mechinales, finalmente desechamos la reproducción de este sistema de forjados por dos razones:

1. El coste de las vigas de madera, de tamaño muy superior al convencional $\left(600 \times 40 \times 30 \mathrm{~cm}^{3}\right)$, excedía a las posibilidades económicas del proyecto, ya fueran en piezas enteras o encoladas.

2. La restauración ha ido avanzando, en los últimos años, en discontinuas y sucesivas fases de bajo presupuesto que inevitablemente se han de realizar con precariedad de medios auxiliares. Por lo tanto, hemos considerado quimérica toda solución constructiva que precisara el trasiego de 


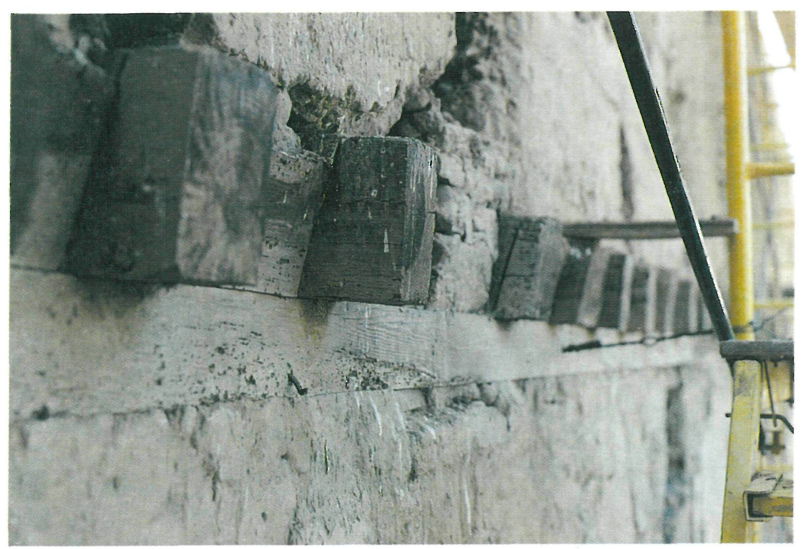

Foto 7. Sistema tradicional de apoyo de forjados de madera.

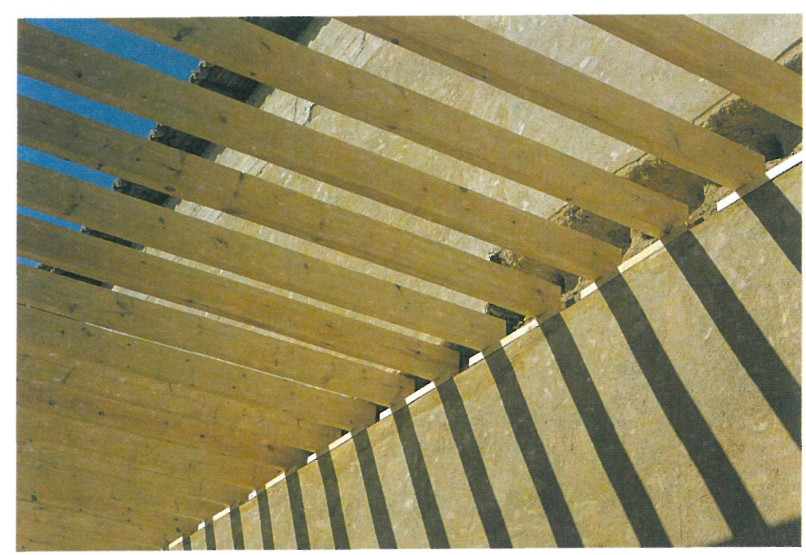

Foto 8. Apoyo del nuevo forjado sobre la roza.

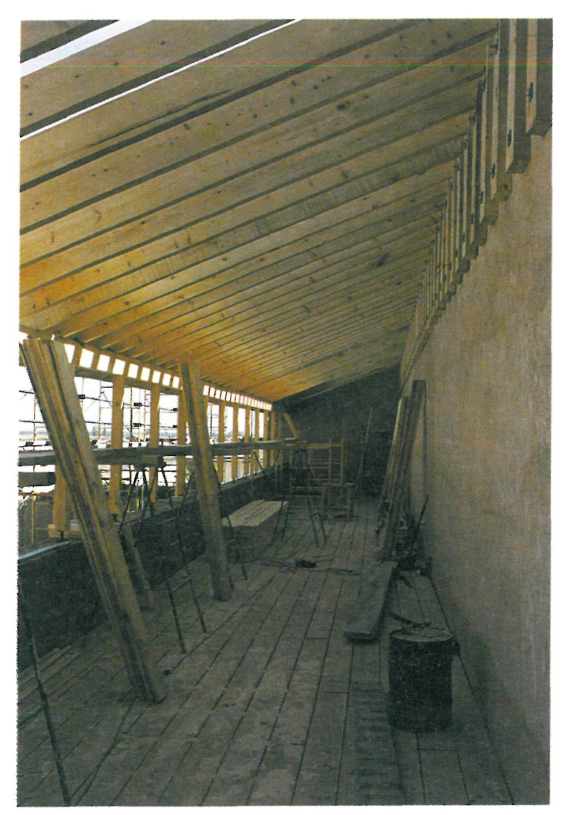

Foto 9. Apoyo de los pares de cubierta sobre los pies adricalos. piezas de peso considerable que, además en este caso, habrían de requerir para su colocación entre dos muros separados por una luz libre inferior a la longitud de éstas de una complicada y penosa maniobra.

En consecuencia, el factor que decantó la elección del tipo de forjado fue el fácil manejo de los componentes conformados de los que estuviera compuesto.

Dentro de la gama de soluciones que cumplen esta exigencia, escogimos la losa unidireccional de hormigón armado de $23+4 \mathrm{~cm}$ de canto, aligerada por un encofrado perdido de piezas de poliestireno expandido de alta densidad.

Encontramos en esta solución las siguientes ventajas:

- Reducción del peso propio en un 25 por ciento respecto de los sistemas convencionales.

- Creación de una envolvente térmica que aísle cada una de las cajas en las que se descompone el espacio de la nave, y que previsiblemente han de funcionar de forma autónoma.

- Disponibilidad en la cara inferior del forjado de una superficie continua de poliestireno de $3 \mathrm{~cm}$ que permite un fácil tendido del cableado de iluminación, habida cuenta sobre todo que la escasez de la altura libre impide la construcción de un cielo raso.

A su vez, hay otra variable que incide en la elección del forjado: la particular naturaleza de la fábrica de tapia con la que están construidos los muros que han de soportarlo.

Conviene a la tapia cualquier solución de forjado que transmita su peso según dos principios básicos: produciendo exclusivamente esfuerzos de compresión y repartiendo su acción lo máximo posible.

Para evitar todo esfuerzo que no fuera la compresión simple desechamos las soluciones que comportaran el uso de modernos medios de anclaje (tacos especiales, etc.) basados en la transmisión por adherencia o por fuertes tracciones locales.

La entrega de la losa se resolvió mediante la apertura en el muro de una roza longitudinal. Es cierto que esta operación cercena la continuidad de la 
protección impermeable de los paramentos; antes de que el agua llegue a infiltrase por esta franja desprotegida será necesaria la ruina previa de la cubierta y de los forjados, es decir una situación de ruina global a la que, sinceramente, no alcanza nuestro natural pesimismo.

Queda por saber cómo se debe realizar la roza, pues en este punto convergen dos circunstancias contradictorias.

De una parte, cuanto más profunda sea ésta, mayor será el plano de asiento y, en consecuencia, menor la tensión local. Sin embargo, también será más arriesgada la situación de descalce en la que queda el trozo de fábrica superior en el lapso de tiempo entre la apertura de la roza y el vertido del hormigón de la losa.

Llegados a este punto conviene hacer unos números.

Con una carga total unitaria de $7,75 \mathrm{kN} / \mathrm{m}^{2}$ y una luz libre media de $5,70 \mathrm{~m}$, cada uno de los dos muros paralelos, que sostienen el forjado, han de soportar por cada metro lineal un peso de $22,10 \mathrm{kN}$.

No parece imprudente otorgar a la fábrica, en el plano de asiento del forjado, una capacidad resistente igual a la que debe soportar en la sección horizontal inferior para sostener su propio peso.

Como la altura máxima del muro es de $12 \mathrm{~m}$ sabemos que la fábrica resiste al menos 12 $\mathrm{m} \times 0,02 \mathrm{mN} / \mathrm{m}^{2} . \mathrm{m}=0,24 \mathrm{mN} / \mathrm{m}^{2}$.

Por tanto, se necesitan 22,10 kN/2,4 mN/m² $=920$ $\mathrm{cm}^{2}$ como superficie de asiento del forjado por cada metro lineal de muro o, lo que es lo mismo, una roza continua de $9 \mathrm{~cm}$ de ancho.

Analizada desde otra perspectiva, la tensión que el peso del forjado aporta al muro, una vez repartido en todo su espesor, es irrelevante. En la fábrica más delgada, que tiene un grosor de $115 \mathrm{~cm}$ equivale a $0,019 \mathrm{mN} / \mathrm{m}^{2}$, es decir menos de lo que aporta el peso propio de una franja de fábrica de un metro de altura.

En consecuencia, el apoyo de las losas se realizó mediante la simple apertura de una roza corrida con forma en sección de pico de flauta y $10 \mathrm{~cm}$ de asiento horizontal (ver Fig. 1) en el que, hasta el momento presente, éstas reposan plácidamente con el añadido de sus correspondientes divisiones, sola-

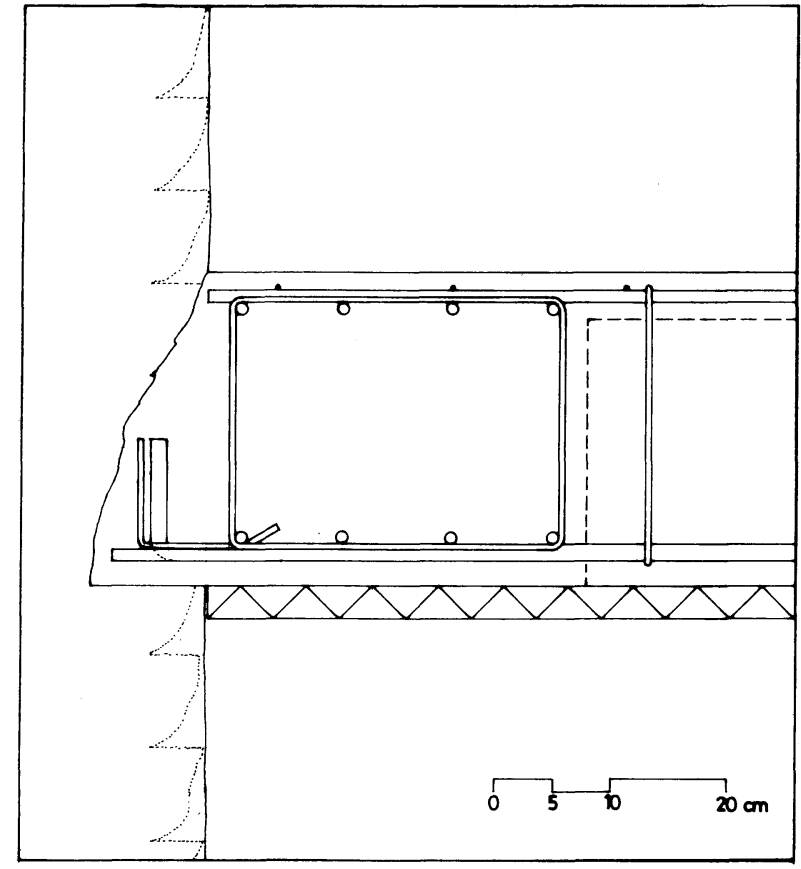

Fig. 1. Apoyo de la losa de hormigón armado.

dos, etc., en contra de los negros auspicios que aquejaron a la dirección técnica cuando, trasladado el plano a la realidad, pudo comprobar lo minúsculos que a veces pueden parecer 10 prosaicos centímetros.

El segundo principio que ha de regir la transmisión de la carga de los forjados a una tapia es el máximo reparto de las acciones. Como ya hemos comentado, esto se debe a la prudencia con la que hay que tratar a una fábrica que no está muy lejos de su tamaño insuperable y que además, en su puesta en obra, puede sufrir episodios muy variados y en general poco favorables.

Esta exigencia se ha satisfecho de tres modos simultáneos:

- La continuidad de la entrega en el muro.

- La disposición de los nervios de la losa con un separación reducida ( $65 \mathrm{~cm}$ entre ejes).

- El remate longitudinal de los bordes de la losa con una viga que garantiza el reparto de las cargas de cada nervio antes del apoyo sobre el muro, contemplando la hipótesis desfavorable de que en éste puedan producirse fallos locales. 


\section{Apoyo sobre un muro de tapia de un forjado de madera}

Dentro del proceso de restitución parcial de la integridad del edificio se ha construido una galería porticada que se adosa a las dos naves, configurando una crujía deambulatoria que desempeña una importante función distribuidora y, a la vez, conforma la imagen del patio interior del Palacio.

Este nuevo elemento arquitectónico se diseñó por analogía respecto del original, esquematizando su geometría y reproduciendo las proporciones y la estratificación de materiales.

En particular, el forjado consta de una única familia de pares de tablón de madera de escuadría convencional, separados $50 \mathrm{~cm}$ a ejes, y un tablero simple de tabloncillo.

El faldón de cubierta es un forjado inclinado idéntico al descrito anteriormente, con una cobertura final de teja cerámica curva.

Al contrario que en el ejemplo precedente, la construcción de los forjados a base de elementos conformados de madera era mucho más viable, pues todas las piezas tenían peso y dimensiones manejables y el apoyo de las viguetas podía ejecutarse sin mayores complicaciones.

La cuestión a resolver era, por tanto, la entrega de un forjado de madera en la cara exterior de un muro de tapia.

Desde nuestro punto de vista el diseño concreto del apoyo había de cumplir una exigencia esencial: conservar intacto el calicostrado, pues aunque esta nueva crujía aleja varios metros el agua de lluvia del paramento en cuestión, la acción de ésta es tan destructiva e inclemente que cualquier rotura de la envolvente impermeable es, a largo plazo, una mácula fatal.

Por ello se diseñó una solución consistente en adosar al muro una carrera paredaña de tablón de madera anclada mediante tacos HSA de la marca "Hilti", uno por cada par, de métrica 12 x 180 en la cubierta y 16 × 220 en el forjado.

El dimensionado de estas piezas se realizó aplicando las disposiciones de la norma EH-91 y atribuyendo a la fábrica de tapia un carácter de hormigón H-30. Esta suposición no es gratuita, sino que está basada en los resultados de los controles de resis- tencia realizados durante la reconstrucción de los muros. En concreto, se estimó una tensión segura a tracción de $0,18 \mathrm{mN} / \mathrm{m}^{2}$ y de 0,14 a cortante.

Desgraciadamente todos estos primorosos cálculos quedaron en barbecho cuando al proceder en el curso de las obras a la sujeción de la carrera comprobamos que un porcentaje significativo de los anclajes, una vez introducidos en la tapia y por causas que todavía desconocemos, no quedaban fijos sino que giraban sin fin.

Hubo, por tanto, que desechar esta solución de apoyo e idear otra con la premura que impone la necesidad de dar ocupación a los trabajadores de la contrata.

Por razones que desarrollaremos posteriormente optamos por dar a forjado y cubierta dos sistemas de apoyo diferentes.

Como ya hemos dicho, esta nueva crujía se adosa a dos muros de tapia perpendiculares entre sí. Uno de ellos fue reconstruido en la primera fase de la restauración, mientras que el otro, salvo la coronación, es original.

En este lienzo antiguo se conservaba la roza horizontal del plano de asiento del forjado primitivo. Esta solución nunca nos pareció muy afortunada, pues vulnera gravemente la durabilidad de la fábrica. A pesar de todo, durante la redacción del Proyecto escogimos, como alternativa más incruenta, reutilizar la roza para apoyar de nuevo la parte del forjado correspondiente.

Lo que nunca imaginábamos es que nos veríamos obligados a estigmatizar del mismo modo el muro vecino pues, ante nuestra incapacidad para conciliar la preservación de la envolvente y la garantía del apoyo, nos decantamos por este segundo factor, habida cuenta de la considerable entidad de las sobrecargas de uso del forjado.

Por supuesto, la transmisión de las cargas de cada vigueta a la fábrica de tapia se resolvió con la mediación de un elemento durmiente que reparte y aminora las compresiones locales.

En el diseño del apoyo de los faldones de cubierta incidían dos factores particulares.

De una parte, la preservación de la continuidad de la envolvente impermeable era un requisito de obligado cumplimiento en un lugar tan expuesto a la acción de los agentes atmosféricos. 


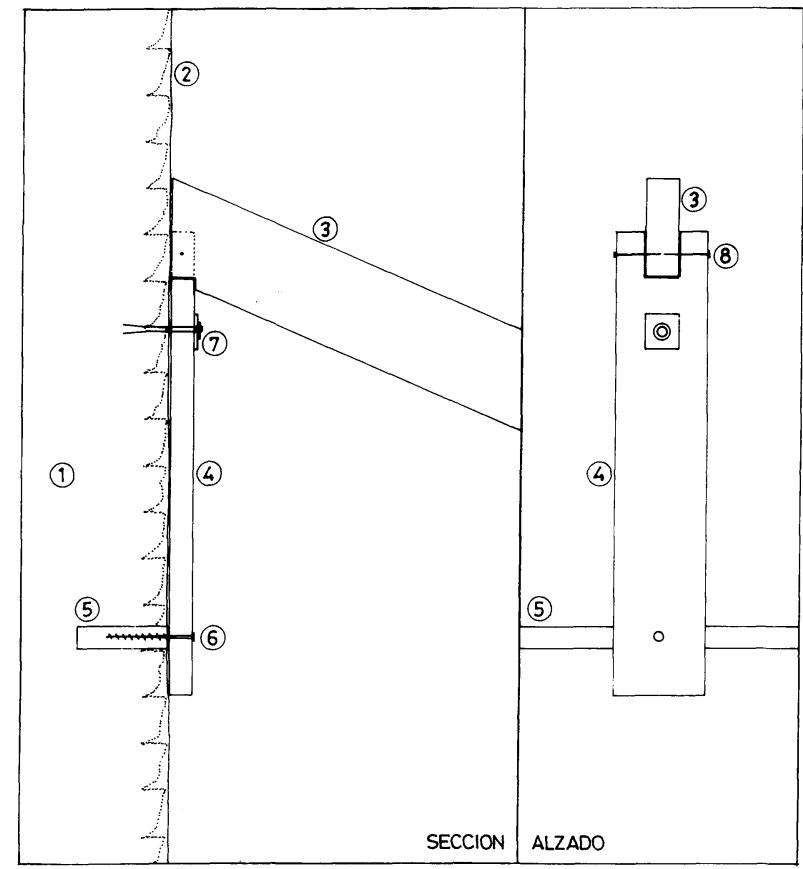

Fig. 2. Apoyo de la cubierta.

1. Fábrica de tapia. 2. Calicostrado. 3. Par. 4. Pie $200 \times 50 \mathrm{~mm}^{2}$. 5. Dur miente $200 \times 50 \mathrm{~mm}^{2}$. 6. Tornillo $180 \mathrm{~mm}$. $\varnothing 15 \mathrm{~mm}$. 7. Taco "Hilti" 8. Dos clavos $100 \mathrm{~mm}$.

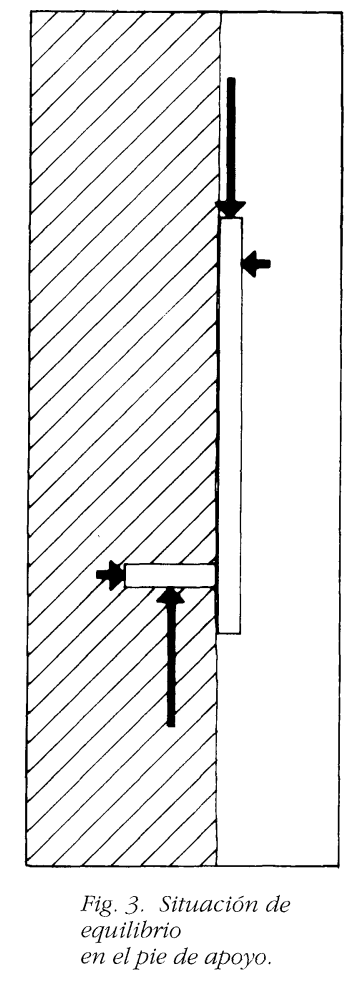

De otra, al suplementar el muro antiguo habíamos dejado embebido longitudinalmente en el paramento de la fábrica un tablón de madera que, a la vez de elemento de sujeción de las puertas de tapiar, servía de límite diferenciador entre lo viejo y lo nuevo. $\mathrm{Al}$ reconstruir el muro colindante decidimos incluir también este apéndice pues, aunque no tenía una utilidad concreta en aquel momento, "para algo habría de servir".

Por una vez el tiempo nos ha dado la razón y la colocación, más intuitiva que reflexiva, de esta durmiente nos ha permitido, seis años después, sostener la cubierta sin cercenar la impermeabilidad del paramento.

Para ello, hemos apoyado cada par en un pequeño pie de tablón de madera anclado a la durmiente mediante un tornillo roscado y a la fábrica con el taco "Hilti" previsto en el proyecto (ver Fig. 2).

La función del taco es auxiliar, pues en principio sólo sirve para bloquear la tendencia al giro del pie de apoyo producido por el par de fuerzas verticales (ver Fig. 3). Aunque la sujeción del taco no es muy fiable, la acción que ha de soportar es muy pequeña, pues el brazo de palanca entre éste y el tornillo es mucho mayor, que el que ha de contrarrestar.

Posiblemente haya sido esta previsión de elementos inútiles una de las más fructíferas enseñanzas de esta obra interminable.
1. Los sucesivos proyectos han sido elaborados y dirigidos por el autor de este artículo con la colaboración de Mariano Vázquez. Espí. Por este motivo el texto está redactado en primera persona del plural.
2. E. Algorri, y M. Vázquez (1991): "La rehabilitación del Palacio de los Guzmanes, León”, en el libro Jornadas sobre Restauración y Conservación de Monumentos, Ministerio de Cultura e Instituto Eduardo Torroja-CSIC. Madrid. 
$\underline{38}$

Informes de la Construcción, Vol. 46, n. ${ }^{\circ}$ 434, noviembre/diciembre 1994

3. I. Paricio Ansuátegui (1985): La construcción de la arquitectura. 2. Los elementos. Barcelona, ITEC.

4. Paricio, op. cit.
5. Para una explicación más prolija ver Algorri y Vázquez, op. cit.

6. Entendido éste no en el sentido moderno de un documento, sino como el conjunto de conceptos manejados por aquel o aquellos que concibieron el edificio.

BIBLIOGRAFÍA BÁSICA

- Craterre (1989): Traité de Construction en Terre, Éditions Parenthèses. Marseille.

— J. Corral (1984): "Sobre el barro y su técnica: la razón natural en los constructores vernáculos", en Arquitectura, técnica y naturaleza en el ocaso de la modernidad, Servicio de Publicaciones del MOPUT. Madrid

- M. Olcense (1993): Arquitecturas de tierra: Tapial y Adobe. Colegio Oficial de Arquitectos. Valladolid.

— E. Algorri y M. Vázquez (1991): "Rehabilitación del Castillo de Toral de los Guzmanes, León”, en el libro Jornadas sobre
Restauración y Conservación de Monumentos. Ministerio de Cultura e Instituto Eduardo Torroja-CSIC. Madrid.

- "Viviendas de muy bajo coste". Informes de la Construcción, números 344-345. Instituto Eduardo Torroja-CSIC. Madrid.

- (1986): "Comprobaciones resistentes de elementos constructivos de tierra”, Informes de la Construcción, número 385. Instituto Eduardo Torroja-CSIC. Madrid.

- "II Concurso Iberoamericano de Informes 1986", Informes de la Construcción, número 386. Instituto Eduardo Torroja-CSIC. Madrid.

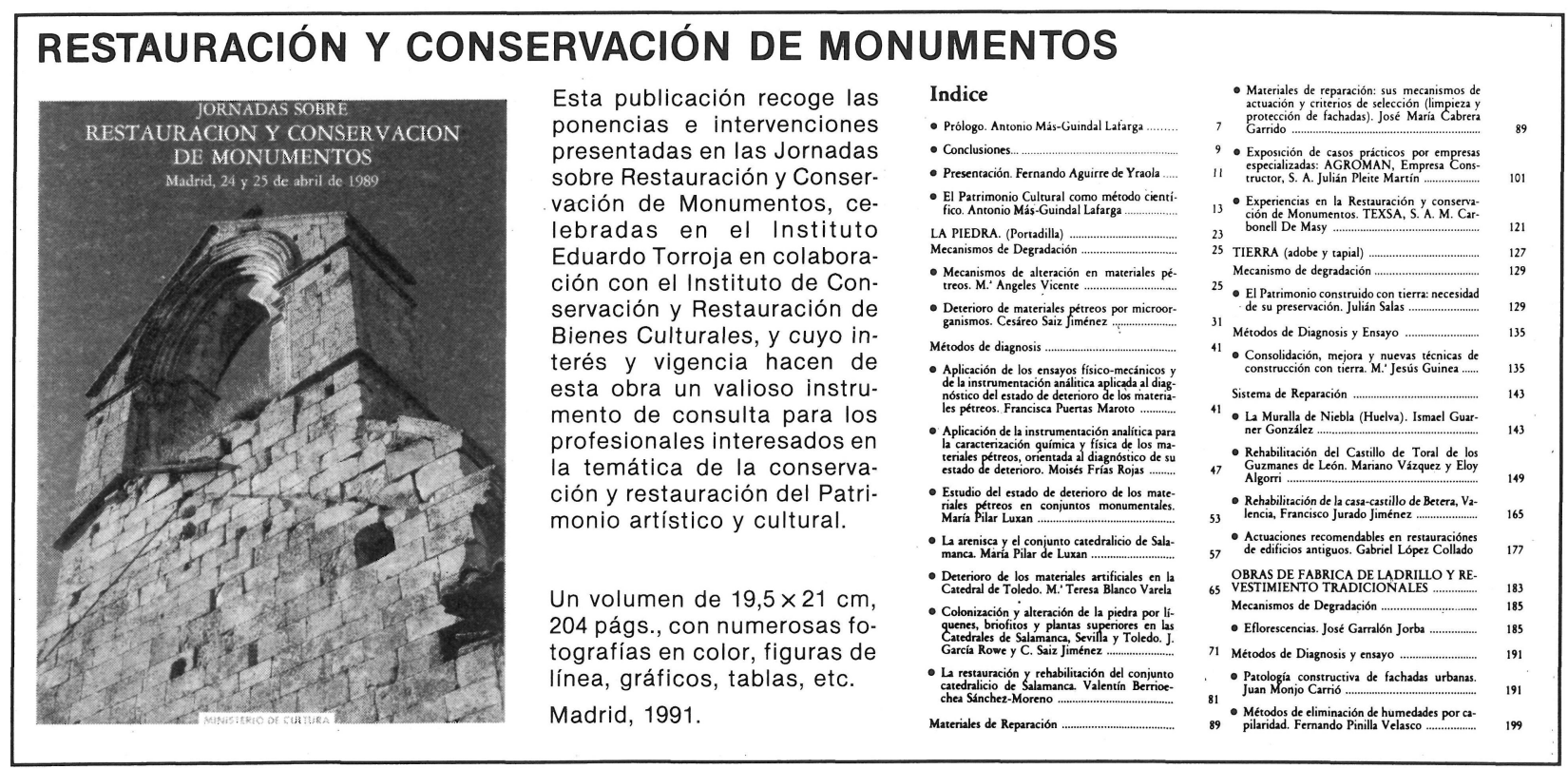

